\title{
Asynchronous optical sampling for high-speed characterization of integrated resonant terahertz sensors
}

\author{
C. Janke, M. Först, M. Nagel, and H. Kurz \\ Institut für Halbleitertechnik, RWTH Aachen University, \\ D-52056 Aachen, Germany \\ A. Bartels \\ Gigaoptics GmbH, D-63165 Mühlheim am Main, Germany
}

\begin{abstract}
Two femtosecond Ti:sapphire lasers with slightly different repetition rates near $1 \mathrm{GHz}$ are coupled to implement high-speed asynchronous optical sampling. The application of this technique is successfully demonstrated in the field of terahertz time-domain spectroscopy (TDS). A time delay of $1 \mathrm{~ns}$ is scanned at a frequency of $5 \mathrm{kHz}$ without moving mechanical parts. Compared with that of conventional TDS schemes based on lock-in detection and moving mirrors, the readout time of integrated resonant $\mathrm{THz}$ sensors is reduced by a factor of 20, opening the way for high-throughput $\mathrm{THz}$ sensing in marker-free DNA analysis.
\end{abstract}

The majority of ultrafast time-resolved measurement techniques have been based on pump-probe schemes in which a single femtosecond (fs) laser system provides both pump and probe pulse trains. Although other techniques have been proposed ${ }^{1-3}$ scanning of the time delay is achieved mostly by mirrors mounted onto motorized translation stages or vibrating membranes. Motorized stages facilitate large time delays, of the order of several hundred picoseconds, but are slow moving and give rise to long scan times. Vibrating membranes operate at scan rates near $100 \mathrm{~Hz}$ and permit significant reductions of data-acquisition times; however, the accessible temporal window is limited because of the small vibration amplitudes. These limitations can be overcome by use of two fs lasers to deliver the pump and probe pulses to the sample.

In such a scheme the time delay is scanned by means of slightly different pulse repetition rates [asynchronous optical sampling ${ }^{4-7}$ (ASOPS)] without the need for moving mechanical parts. Unlike in a related method, ${ }^{8}$ the repetition rates are not modulated but are stabilized with a fixed difference. The available measurement window is given by the pulse-topulse spacing (i.e., the inverse of the repetition rate), whereas the scan rate is determined solely by the repetition rate detuning. This detuning can be set to any value from a few hundred hertz to several megahertz, providing the means for a major reduction in measurement time. An upper limit to the scan rate is imposed by the available bandwidth of the detection and data-acquisition electronics and the desired time resolution. The relation between time resolution and scan rate becomes increasingly favorable if laser systems with gigahertz repetition rates are used. Inherent advantages of ASOPS compared with standard pump-probe techniques based on a single fs laser are the absence of changes in optical spot size at long time delays, the absence of pointing instabilities, and the elimination of noise caused by moving mechanical components. In addition, the performance of twocolor experiments is straightforward, providing further versatility.

We employ two Ti:sapphire fs lasers with repetition rates near $1 \mathrm{GHz}$ to achieve high-speed implementation of ASOPS and have successfully demonstrated its application in the field of $\mathrm{THz}$ time-domain spectroscopy. The readout time of integrated resonant $\mathrm{THz}$ sensors designed for biomolecular sensing is significantly reduced compared with that of conventional lock-in detection based on moving mirrors. Arrays of these sensors have been shown to be efficient tools for marker-free on-chip detection of biomolecules in the terahertz (THz) frequency range. ${ }^{9}$

To facilitate asynchronous optical sampling, we require the fs lasers to operate at slightly different repetition rates, $f_{r, 1}$ and $f_{r, 2}$. For notational purposes only, laser 1 is designated the pump laser and laser 2 the probe laser. The time delay between the pump and the probe pulses is scanned at a frequency $\Delta f_{r}$ $=f_{r, 2}-f_{r, 1}$. For a desired time resolution $\tau$ at a given usable detection bandwidth $F$ (determined by the detection and data-acquisition hardware), $\Delta f_{r}$ is restricted according to ${ }^{10} \Delta f_{r} \leqslant \tau f_{r, 2} F$. Accordingly, $\Delta f_{r}$ scales linearly with $f_{r .2}$. If $\tau$ and $F$ are taken to be fixed, the benefits of ASOPS are thus best exploited if high-repetition-rate lasers are used. With a pump laser repetition rate $f_{r, 1}=1 \mathrm{GHz}$, the available temporal measurement window is $f_{r, 1}{ }^{-1}=1 \mathrm{~ns}$. This is distinctly larger than what is typically accessible with motorized translation stages and well suited for the investigation of long-lived transient phenomena as en- 
countered in $\mathrm{THz}$ gas spectroscopy and in picosecond ultrasonics, ${ }^{11,12}$ for example.

The experimental setup is sketched in Fig. 1. It consists of two fs lasers with repetition rates close to $1 \mathrm{GHz}$ (Ref. 13; Model GigaJet 20 from Gigaoptics $\mathrm{GmbH}$ ) and the integrated resonant $\mathrm{THz}$ sensor as main building blocks. The sensor comprises two photoconductive switches (PC) for pulsed generation and detection of $\mathrm{THz}$ signals in a pump-probe scheme. ${ }^{14}$ Electrical subpicosecond pulses are optically generated at one of the switches biased at $+20 \mathrm{~V}$. They subsequently travel along thin-film microstrip lines and are transmitted through the $\mathrm{THz}$ ring resonator. Time-delayed optical probe pulses gate the second photoconductive switch and allow for time-resolved detection of the transient photocurrent generated by the incident $\mathrm{THz}$ field. To obtain the trigger signal required for the data-acquisition system, we split off a small amount of optical power from both pulse trains. A cross-correlation signal, based on noncollinear second-harmonic generation in a nonlinear $\mathrm{LiIO}_{3}$ crystal, is generated at $\Delta f_{r}$ and detected with a photodiode.

Fluctuations in the repetition rate of each laser lead to a deviation from the chosen scan rate $\Delta f_{r}$, which gives rise to a timing jitter with respect to the nominal time delay $T=\Delta f_{r} t / f_{r, 1}$, with real time $t$. To reduce this jitter we actively stabilize the cavity lengths of both lasers with piezo-driven mirrors. The piezoelectric transducers are controlled by two phase-locked loops (PLLs) that share a common time base, which serve to lock the repetition rates to external reference oscillators. The timing stability is characterized by use of a second noncollinear secondharmonic generation setup. The temporal width of the cross-correlation signal between the two lasers is $\simeq 1 \mathrm{ps}$ within the measurement window of $1 \mathrm{~ns}$. Although it is substantially larger than the 50 -fs pulse duration of the fs lasers, this stability is more than sufficient, as discussed below. In addition, the dualPLL scheme used here yields better stability than locking beat frequency $\Delta f_{r}$ with a single PLL.

Figure 2(a) shows the temporal evolution of an electrical input pulse transmitted through the $\mathrm{THz}$ resonator as mapped by the ASOPS technique. At a scanning frequency of $\Delta f_{r}=5 \mathrm{kHz}$ this signal is ob-

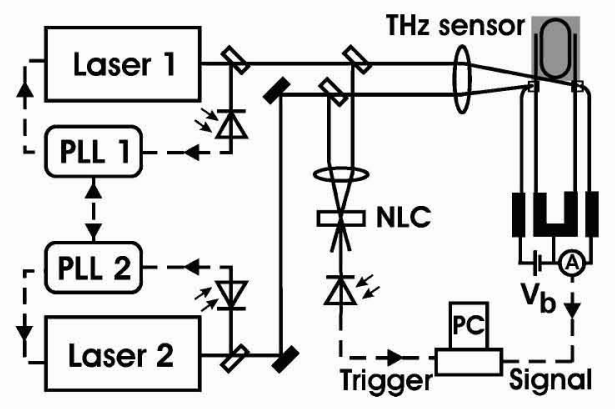

Fig. 1. Experimental setup that comprises two fs lasers at a $1-\mathrm{GHz}$ repetition rate and an integrated resonant $\mathrm{THz}$ sensor. The repetition rates are locked to two synthesizers, sharing a common time base by use of two PLLs. The trigger signal for the data-acquisition system is generated by use of a nonlinear $\mathrm{LiIO}_{3}$ crystal (NLC).
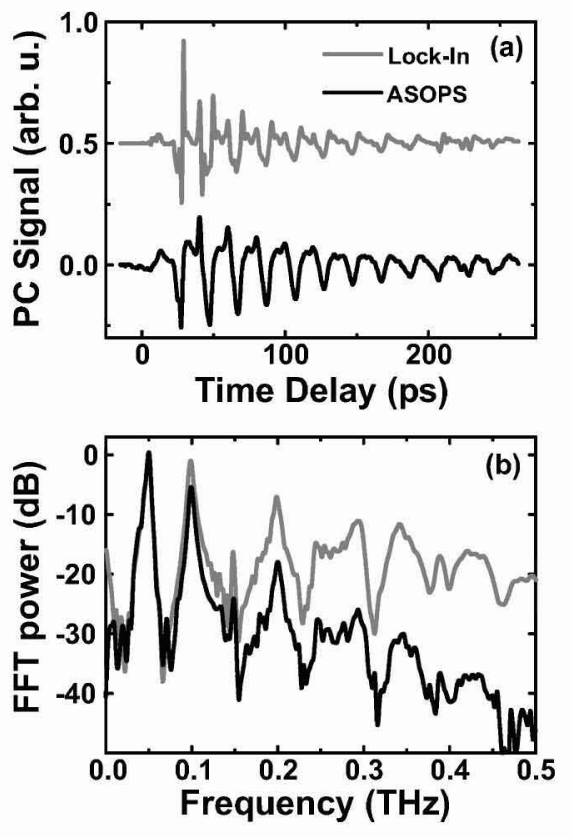

Fig. 2. (a) Time-domain transmission signal as mapped by ASOPS at $\Delta f_{r}=5 \mathrm{kHz}(250,000$ scans, darker curve) and by a standard lock-in detection scheme based on moving mirrors (single scan, lighter curve). The transients are offset vertically for clarity. (b) Corresponding Fourier-transform spectra (normalized).

tained by averaging of 250,000 individual scans with a remarkably short acquisition time of only $50 \mathrm{~s}$. It exhibits an exponentially damped beating of different frequency components, which are determined by the transmission characteristics of the $\mathrm{THz}$ resonator. Backreflections of the signal from open-ended waveguides of the device structure limit the accessible measurement window to approximately $300 \mathrm{ps}^{9}$ For comparison, the lighter curve represents a standard lock-in measurement (single scan; time constant, $200 \mathrm{~ms}$ ) by use of a motorized translation stage with an overall acquisition time of $15 \mathrm{~min}$. Note that, unlike in the lock-in technique, scanning the full delay of $1 \mathrm{~ns}$ with ASOPS does not increase the acquisition time.

To provide insight into the spectral transmission characteristics, Fig. 2(b) shows the corresponding Fourier-transform spectra of the acquired timedomain data. For frequencies up to $0.4 \mathrm{THz}$, both spectra exhibit pronounced local maxima at integer multiples of $50 \mathrm{GHz}$. These resonance frequencies are in excellent agreement with theoretically predicted values with the average circumference of the resonator and the effective permittivity of the waveguides taken into account. ${ }^{9}$ The resonances' quality factor is defined by $Q=\nu_{r} / \Delta \nu_{r}$, where $\nu_{r}$ is the resonance frequency and $\Delta \nu_{r}$ is the corresponding 3-dB bandwidth. The values obtained by high-speed ASOPS are $Q(50 \mathrm{GHz})=11.1, \quad Q(99 \mathrm{GHz})=18.0$, $Q(149 \mathrm{GHz})=38.7$, and $Q(200 \mathrm{GHz})=20.6$. For lock-in detection $Q(50 \mathrm{GHz})=10.8, Q(99 \mathrm{GHz})=16.7$, $Q(149 \mathrm{GHz})=45.2$, and $Q(200 \mathrm{GHz})=19.7$. Hence the spectral position and quality factors of the resonances measured with high-speed ASOPS at a 
greatly reduced acquisition time match those obtained with the conventional lock-in technique.

Neither the jitter with respect to nominal delay $T$ nor the $100-\mathrm{MHz}$ sampling rate of the dataacquisition hardware constrains the time resolution and thus the usable bandwidth of this high-speed ASOPS approach. The transimpedance amplifier that amplifies and converts the transient photocurrent into a voltage signal, however, provides a detection bandwidth of only $F=1.8 \mathrm{MHz}$ at the required gain setting of $10^{6} \mathrm{~V} / \mathrm{A}$. Because in this investigation only frequency components up to $\simeq 0.4 \mathrm{THz}$ are of interest, a time resolution of $\simeq 2.5 \mathrm{ps}$ is sufficient and can be achieved by setting $\Delta f_{r}=5 \mathrm{kHz}$. Nonetheless, the limitation of $F$ manifests itself in a decrease in spectral amplitude toward higher frequencies, as one can see from Fig. 2(b).

To demonstrate the potential of ASOPS in the area of $\mathrm{THz}$ biosensing, ${ }^{9}$ we altered the dielectric properties of the $\mathrm{THz}$ sensor by covering the resonator (shaded area in Fig. 1) with a spin-on polymer. The thickness of the polymer layer (AZ 5214 photoresist) was approximately $1.3 \mu \mathrm{m}$. Figure 3 shows the ASOPS data obtained with and without polymer coating at $\Delta f_{\text {rep }}=5 \mathrm{kHz}$ and 250,000 scans. As is clearly resolved in the time domain (Fig. 3 inset) the coating increases the resonator losses and detunes the resonant features owing to an increase in refractive index. Accordingly, the spectral amplitude is reduced and the resonances are shifted toward lower frequencies, as shown in Fig. 3. The relative frequency shift is $\Delta \nu_{\text {rel }}=1.25 \mathrm{GHz}$, or $2.5 \%$. Even smaller frequency shifts usually encountered in $\mathrm{THz}$ biosensing could be detected with sufficient signal strength. Together with the quality factors of the sensor's resonances, this result demonstrates the potential of high-speed ASOPS for marker-free biosensing applications.

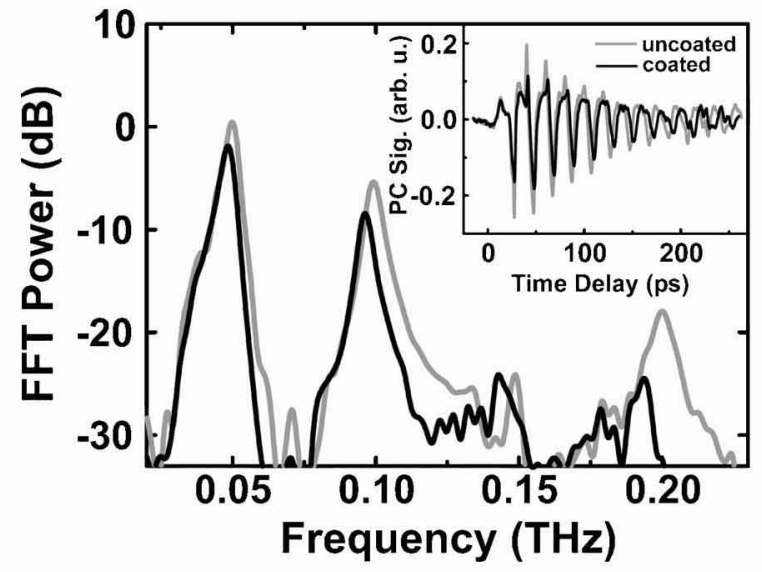

Fig. 3. Inset, time-domain data with and without a polymer coating on the resonator structure. Larger figure, corresponding fast-Fourier-transform (FFT) spectra (normalized).
In conclusion, high-speed asynchronous optical sampling with two stabilized fs lasers with slightly different repetition rates near $1 \mathrm{GHz}$ has been demonstrated in the area of $\mathrm{THz}$ time-domain spectroscopy. This approach facilitates the acquisition of transient pump-probe signals at significantly increased scan rates and provides access to a 1-ns measurement window. Further inherent advantages are the absence of pointing instabilities and spot-size variations from the sample, the elimination of mechanical noise, and added versatility for two-color schemes. In all-optical $\mathrm{THz}$ experiments, such as electro-optic generation and detection of $\mathrm{THz}$ radiation, transimpedance amplifiers are obsolete, and the full 100$\mathrm{MHz}$ bandwidth of the data-acquisition system can be used. With adequate timing stabilization, an unprecedented scan rate of $100 \mathrm{kHz}$ at a time resolution of $100 \mathrm{fs}$ can thus be achieved. Hence the research reported here represents an important step toward high-throughput biomolecular analytics by use of integrated $\mathrm{THz}$ sensor arrays.

We thank J. Martini and M. Hessing for providing the data-acquisition system and gratefully acknowledge previous research by F. Recht. Financial support was provided by the Deutsche Forschungsgemeinschaft. M. Först's e-mail address is foerst@iht.rwthaachen.de.

\section{References}

1. K. F. Kwong, D. Yankelevich, K. C. Chu, J. Heritage, and A. Dienes, Opt. Lett. 18, 558 (1993).

2. M. Lai, Appl. Opt. 40, 6334 (2001).

3. J. Xu and X.-C. Zhang, Opt. Lett. 29, 2082 (2004).

4. P. A. Elzinga, R. J. Kneisler, F. E. Lytle, Y. Jiang, G. B. King, and N. M. Laurendeau, Appl. Opt. 26, 4303 (1987).

5. R. J. Kneisler, F. E. Lytle, G. J. Fiechtner, Y. Jiang, G. B. King, and N. M. Laurendeau, Opt. Lett. 14, 260 (1989).

6. J. D. Kafka, J. W. Pieterse, and M. L. Watts, Opt. Lett. 17, 1286 (1992).

7. S. Adachi, A. Takeyama, Y. Takagi, A. Tackeuchi, and S. Muto, Appl. Phys. Lett. 68, 964 (1996).

8. G. Sucha, M. E. Fermann, D. J. Harter, and M. Hofer, IEEE J. Sel. Top. Quantum Electron. 2, 605 (1996).

9. M. Nagel, F. Richter, P. Haring Bolivar, and H. Kurz, Phys. Med. Biol. 48, 3625 (2003).

10. S. Adachi, S. Takeyama, and Y. Takagi, Opt. Commun. 117, 71 (1995).

11. S. A. Harmon and R. A. Cheville, Appl. Phys. Lett. 85, 2128 (2004).

12. H. T. Grahn, H. J. Maris, and J. Tauc, IEEE J. Quantum Electron. 25, 2562 (1989).

13. A. Bartels, T. Dekorsy, and H. Kurz, Opt. Lett. 24, 996 (1999).

14. T. Pfeifer, H. M. Heiliger, T. Löffler, C. Ohlhoff, C. Meyer, G. Lüpke, H. G. Roskos, and H. Kurz, IEEE J. Sel. Top. Quantum Electron. 2, 586 (1996). 